\title{
Separable Programming to a Multivariate Allocation Problem
}

\author{
Kaynat Nasser \\ Ph.D. (Statistics) \\ Assistant Professor \\ College of Engineering, Teerthankar Mahaveer \\ University, \\ Moradabad, India
}

\author{
Q.S. Ahmad \\ Ph.D. (Operation Research) \\ Associate Professor \\ Department of Mathematics, Integral University, \\ Lucknow, India
}

\begin{abstract}
In this paper the multivariate allocation problem with upper limits on the available costs for various characters is considered. This problem is formulated as a separable programming problem and then solving it by separable programming approach. A numerical illustration is also given.
\end{abstract}

\section{Keywords}

Multivariate allocation problem, non-linear programming, separable programming

\section{INTRODUCTION}

The problem of sample allocation in multivariate stratified sampling has drawn the attention of the researchers for a long time starting apparently with Neyman (1934) [1]. Several criteria of allocation are put forward in the literature. For example, Ghosh (1958) [2] has considered an allocation based on the minimization of generalized variance. Yates (1960) [3] has suggested a procedure in which the cost is minimized for given precision for the estimate of each characteristic. Cochran (1963) [4] suggested the use of the average of individual optimum allocation for various characters. Kokan and Khan (1967) [5] gave an analytical solution to the above problem. Rao, T. J. (1993) [6] has reviewed many of the available criteria of allocation and also suggested new procedures.

We consider the multivariate allocation problem when the costs of enumerating the different characters have been fixed as upper limits. The objectives to be minimized are the variances of the estimates for various characteristics. The problem is formulated as a non-linear programming problem with linear objective function and several non- linear convex constraints, which is of separable nature. In this paper, an approximate solution to this problem is obtained by using separable programming technique.

\section{STATEMENT OF THE PROBLEM}

Consider a multivariate population partitioned into $L$ strata. Suppose that $p$ characteristics are measured on each unit of the population. We assume that the strata boundaries are fixed in advance. Let $n_{i}$ be the number of units drawn without replacement from $i^{t h}$ stratum $(i=1,2, \ldots, L)$. Let $N_{i}$ be the size of the $i^{\text {th }}$ stratum.For $j^{\text {th }}$ character, an unbiased estimate of the population mean $\bar{Y}_{j}(j=1,2, \ldots, p)$, denoted by $\bar{y}_{j s t}$ has its sampling variance

$V\left(\bar{y}_{j s t}\right)=\sum_{i=1}^{L}\left(\frac{1}{n_{i}}-\frac{1}{N_{i}}\right) W_{i}^{2} S_{i j}^{2}$

Where $\quad W_{i}=\frac{N_{i}}{N}, S_{i j}^{2}=\frac{1}{N_{i}-1} \sum_{k=1}^{N_{i}}\left(y_{i j k}-\bar{Y}_{i j}\right)^{2}$

Substituting $a_{i j}=W_{i}^{2} S_{i j}^{2}$, we get

$V\left(\bar{y}_{j s t}\right)=\sum_{i=1}^{L} \frac{a_{i j}}{n_{i}}-\sum_{i=1}^{L} \frac{a_{i j}}{N_{i}}$

Let $C_{i j}$ be the cost of enumerating the $j^{\text {th }}$ character in the $i^{\text {th }}$ stratum and let $C_{j}$ be the amount received for $j^{\text {th }}$ character. Then assuming linear cost function, the cost of the survey may be expressed $\sum_{i=1}^{L} C_{i j} n_{i} \leq C_{j}$,

$$
j=1,2, \ldots, p
$$

Further, the survey is to be conducted in such a way that the variances for all the $p$ characteristics are minimized.

$$
\sum_{i=1}^{L} \frac{a_{i j}}{n_{i}}, \quad\left(N_{i}^{s} \text { are given }\right)
$$

Where $\quad a_{i j}=W_{i}^{2} S_{i j}^{2}$,

Using the transformation $\frac{1}{n_{i}}=x_{i}$, the allocation problem reduces to the following multi-objective convex programming

$$
\begin{aligned}
& \text { Minimize } V_{j}=\sum_{i=1}^{L} a_{i j} x_{i} \text {, } \\
& \text { problem } \\
& \left.\begin{array}{rl}
\text { Subject to } \sum_{i=1}^{L} \frac{C_{i j}}{x_{i}} \leq C_{j}, & j=1,2, \ldots, p \\
\frac{1}{N_{i}} \leq x_{i} \leq 1, & i=1,2, \ldots, L
\end{array}\right\}
\end{aligned}
$$

As both the objective function and the constraints are separable, it is possible to write the problem (2.3) in the form of a separable programming problem as follows: 
Minimize $\sum_{i=1}^{L} f_{i j}\left(x_{i}\right)$,

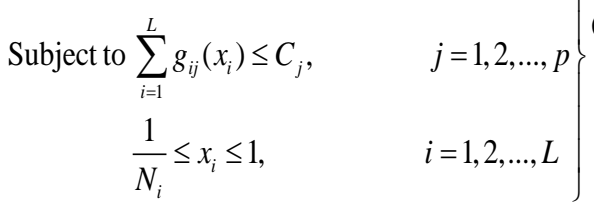

Where $f_{i j}\left(x_{i}\right)=a_{i j} x_{i}$,

$g_{i j}\left(x_{i}\right)=\frac{C_{i j}}{x_{i}}, \quad i=1,2, \ldots, L$

\section{SOLUTION USING SEPARABLE PROGRAMMING}

The non-linear functions $g_{i j}\left(x_{i}\right)$ can be approximated by piecewise linear functions. Let the feasible range of the variables be $x_{i}$, given by the interval $\left[\alpha_{i}, \beta_{i}\right]$ and choose a set of $m_{i}$ grid points $\alpha_{i r}\left(r=1,2,, \ldots, m_{i}\right)$ such that $\alpha_{i}=\alpha_{i 1}<\alpha_{i 2}<\ldots<\alpha_{i m_{i}}=\beta_{i}$.

Every point $x_{i}$ in the grid $\left[\alpha_{i r}, \alpha_{i r+1}\right]$ can be expressed as

$x_{i}=\lambda_{i r} \alpha_{i r}+\lambda_{i, r+1} \alpha_{i, r+1}$

Where $\quad \lambda_{i r}+\lambda_{i, r+1}=1$

and $\lambda_{i r} \geq 0, \lambda_{i, r+1} \geq 0$

A linear approximation for a function $g_{i j}\left(x_{i}\right)$ in the grid interval is $\quad\left[\alpha_{i r}, \alpha_{i r+1}\right]$ is $\widehat{g}_{i j}\left(x_{i}\right)=\lambda_{i r} g_{i j}\left(\alpha_{i r}\right)+\lambda_{i, r+1} g_{i j}\left(\alpha_{i, r+1}\right)$. In general for $x_{i} \varepsilon\left[\alpha_{i}, \beta_{i}\right]$, the piecewise linear approximation $\widehat{g}_{i j}\left(x_{i}\right)$ can be written as

$\widehat{g}_{i j}\left(x_{i}\right)=\sum_{r=1}^{m_{i}} \lambda_{i r} g_{i j}\left(\alpha_{i r}\right)$

With $x_{i}=\sum_{r=1}^{m_{i}} \lambda_{i r} \alpha_{i r}, \sum_{r=1}^{m_{i}} \lambda_{i r}=1 ; \lambda_{i r} \geq 0$,

For $r=1,2, \ldots, m_{i}$

Provided for each, $i$ at the most two adjacent $\lambda_{i r}$ are positive.

An approximating linear program to the non-linear separable program (2.4) is thus obtained as

$$
\left.\begin{array}{rl}
\text { Minimize } Z & =\sum_{i=1}^{L} \sum_{r=1}^{m_{i}} \lambda_{i r} g_{i j}\left(\alpha_{i r}\right) \\
\text { Subject to } \sum_{i=1}^{L} \sum_{r=1}^{m_{i}} \lambda_{i r} g_{i j}\left(\alpha_{i r}\right) \leq C_{j}, & j=1,2, \ldots, p \\
\sum_{r=1}^{m_{i}} \lambda_{i r}=1, \lambda_{i r} \geq 0, & r=1,2, \ldots, m_{i}
\end{array}\right\}
$$

Problem (3.3) is a linear programming problem and can be solved by simplex method using restricted basis entry rule for separable functions Hadley (1964)[7]. Theoptimal values of $\lambda_{i r}^{*}\left(r=1,2, \ldots, m_{i} \& i=1,2, \ldots, L\right)$ obtained by solving problem (3.3) yield an approximate optimal solution $\hat{x}$ to the original problem (2.4) as

$$
\widehat{x}_{i}=\sum_{r=1}^{n_{i}} \lambda_{i r}^{*} \alpha_{i r}, \quad i=1,2, \ldots, L
$$

Example: Consider an allocation problem with one character $(p=1) \&$ two strata $(L=2)$, with following information:

\begin{tabular}{|l|l|l|l|l|l|l|}
\hline$i$ & $W_{i}$ & $S_{i 1}$ & $S_{i 2}$ & $C_{i 1}$ & $C_{i 2}$ & $N_{i}$ \\
\hline 1 & 0.3 & 2 & 3 & 0.6 & 1.5 & 18 \\
\hline 2 & 0.45 & 4 & 1 & 0.8 & 2 & 27 \\
\hline
\end{tabular}

The variance coefficients matrix is given by:

$$
\left(a_{i j}\right)=\left(\begin{array}{ll}
0.36 & 0.81 \\
3.24 & 0.2025
\end{array}\right)
$$

Let us fix the budget at 80 and 100 units.

Using the above information the problem in (2.3) will be as follows:

Thus,

$$
\begin{gathered}
\text { s.t. } \frac{0.6}{x_{1}}+\frac{0.8}{x_{2}} \leq 80 \\
\frac{1.5}{x_{1}}+\frac{2}{x_{2}} \leq 100 \\
0.055 \leq x_{1} \leq 1 \\
0.037 \leq x_{2} \leq 1
\end{gathered}
$$$$
\text { Min. } V=1.17 x_{1}+3.4425 x_{2}
$$

$\alpha_{11}=0.055, \alpha_{12}=0.2912, \alpha_{13}=0.5275, \alpha_{14}=0.7367, \alpha_{15}=1$ $\alpha_{21}=0.055, \alpha_{22}=0.2912, \alpha_{23}=0.5275, \alpha_{24}=0.7367, \alpha_{25}=1$

The piecewise linear approximation to the function $f_{1}\left(x_{1}\right)=1.17 x_{1}, f_{2}\left(x_{2}\right)=3.4425 x_{2}$

$g_{11}\left(x_{1}\right)=\frac{0.6}{x_{1}}, g_{12}\left(x_{2}\right)=\frac{0.8}{x_{2}}, g_{21}\left(x_{1}\right)=\frac{1.5}{x_{1}}, g_{22}\left(x_{2}\right)=\frac{2}{x_{2}} \quad$ are $\hat{f}_{1}\left(x_{1}\right)=0.064 \lambda_{11}+0.341 \lambda_{12}+0.617 \lambda_{13}+0.862 \lambda_{14}+1.17 \lambda_{15}$ $\hat{f}_{2}\left(x_{2}\right)=0.189 \lambda_{21}+1.003 \lambda_{22}+1.816 \lambda_{23}+2.536 \lambda_{24}+3.443 \lambda_{25}$ $\hat{g}_{11}\left(x_{1}\right)=10.909 \lambda_{11}+2.060 \lambda_{12}+1.137 \lambda_{13}+0.814 \lambda_{14}+0.6 \lambda_{15}$ $\hat{g}_{12}\left(x_{2}\right)=14.545 \lambda_{21}+2.747 \lambda_{22}+1.517 \lambda_{23}+1.086 \lambda_{24}+0.8 \lambda_{25}$ $\hat{g}_{21}\left(x_{1}\right)=27.273 \lambda_{11}+5.151 \lambda_{12}+2.844 \lambda_{13}+1.964 \lambda_{14}+1.5 \lambda_{15}$ $\hat{g}_{22}\left(x_{2}\right)=36.364 \lambda_{21}+6.868 \lambda_{22}+3.791 \lambda_{23}+2.619 \lambda_{24}+2.0 \lambda_{25}$

Thus the approximated separable programming problem becomes

$$
\begin{array}{cc}
\text { Minimize } & \hat{f}_{1}\left(x_{1}\right)+\hat{f}_{2}\left(x_{2}\right) \\
\text { s.t. } & \hat{g}_{11}\left(x_{1}\right)+\hat{g}_{12}\left(x_{2}\right) \leq 80 \\
\hat{g}_{21}\left(x_{1}\right)+\hat{g}_{22}\left(x_{2}\right) \leq 100 \\
\lambda_{11}+\lambda_{12}+\lambda_{13}+\lambda_{14}+\lambda_{15}=1 \\
\lambda_{21}+\lambda_{22}+\lambda_{23}+\lambda_{24}+\lambda_{25}=1
\end{array}
$$


Solving the above linear programming problem by simplex method using restricted basis entry rule. The optimal solution is given by

$$
\begin{aligned}
& \lambda_{11}=1, \lambda_{12}=0, \lambda_{13}=0, \lambda_{14}=0, \lambda_{15}=0 \\
& \lambda_{21}=1, \lambda_{22}=0, \lambda_{23}=0, \lambda_{24}=0, \lambda_{25}=0
\end{aligned}
$$

Thus, $\quad x_{1}=\sum_{r=1}^{5} \lambda_{1 r} \alpha_{1 r}=0.055$

$$
x_{2}=\sum_{r=1}^{5} \lambda_{2 r} \alpha_{2 r}=0.055
$$

With Min. $Z=0.2537$

\section{CONCLUSION}

In this particular example the optimal solution for the varying intervals(3,5 and 9) is same i.e., MinimumZ $=0.2537$ with $x_{1}=0.055$ and $x_{2}=0.055$

\section{FURTHER STUDY}

This can be checked for other examples whether the no. of intervals make any difference to the optimality of the solution or not and if us then how many no. of intervals.

\section{REFERENCES}

[1] Neyman, J. (1934). On the two different aspects of representative method: The method of stratified sampling and the method of purposive selection. Jour Roy. Stas. Soc., 97, 558-606.

[2] Ghosh, S. P. (1958). A note on stratified random sampling with multiple characters. Cal. Stat. Assoc. Bull., 8, 81-89.

[3] Yates, F. (1960). Sampling methods for censuses and surveys ( $2^{\text {nd }}$ ed). Charles Griffin and Co. Ltd. London.

[4] Cochran, W.G. .(1977). Sampling Techniques. John Wiley \& Sons, New York.

[5] Kokan, A.R.and Khan, S.U. (1967). Optimum Allocation in Multivariate Surveys-An Analytical Solution. Jour. Roy. Stat. Soc. Ser. B.29, 115-125.

[6] Rao, T.J. (1993). On certain problems of sampling designs and estimation for multiple characteristics. Sankhya B, 55, 372-384.
[7] Hadley, G. (1964). Non Linear and Dynamic Programming. Addison - Wesley, publishing company, London.

[8] Pirzada, S. And Maqbool, S. (2003). Optimal Allocation in Multivariate Sampling Through Chebyshev Approximation. Bull. Malaysian Math. Sc. Soc. (Second Series) 26, 221-230

[9] Morris, H. Hansen, et. Al. (1951) Response errors in surveys. JASA, Vol. 46, No. 254, 147-190.

[10] Khan, S. (1971). Minimizing a homogeneous separable function constrained by linear inequalities. Aligarh Bulletin of Maths. 1, 63-71.

[11] Bazaraa, M.S., Sherali, H.D. and Shetty, C.M. (2006) Nonlinear Programming: Theory and Algorithms. John Wiley and Sons, New York, Third Edition.

[12] Day,C.D.(2010).A Multi-Objective Evolutinary Algorithm for Multivariate Optimal Allocation, Section on Survey Research Methods - JSM.

[13] Garciá, J.A.D. and Cortez, L.U. (2006). Optimum Allocation in Multivariate Stratified Sampling: MultiObjective Programming, Comunicaciones Del Cimat, no I-06-07.

[14] Khan, M. F., Ali I. and Ahmad, Q.S. (2011). Chebyshev Approximate Solution to Allocation Problem in Multiple Objective Surveys with Random Costs, American Journal of Computational Mathematics, 1, pp. 247-251.

[15] Kozak, M. (2006) . Multivariate Sample Allocation: Application of Random Search Method, Statistics in Transition, 7 (4), pp. 889-900.

[16] Mohd. Vaseem Ismail , Kaynat Nasser , Qazi Shoeb Ahmad. (2010). Multi-objective convex programming problem arising in multivariate sampling," International Journal of Engineering, Science and Technology Vol. 2, No. 6, 2010, pp. 291-296.

[17] M. Khan, I. Ali, Y. Raghav and A. Bari, (2012). Allocation in Multivariate Stratified Surveys with NonLinear Random Cost Function, American Journal of Operations Research, Vol. 2 No. 1, pp. 100-105. 